\title{
BMJ
}

\section{Intrauterine exposure to carbamazepine and specific congenital malformations: systematic review and case- control study}

\begin{abstract}
Janneke Jentink, research fellow and lecturer in pharmacoepidemiology, ${ }^{1}$ Helen Dolk, professor of epidemiology and health services research, ${ }^{2}$ Maria A Loane, EUROCAT research fellow and lecturer in public health, ${ }^{2}$ Joan K Morris, professor of medical statistics, ${ }^{3}$ Diana Wellesley, clinical geneticist, ${ }^{4}$ Ester Garne, consultant paediatrician and EUROCAT perinatal epidemiologist, ${ }^{5}$ Lolkje de Jong-van den Berg, professor in pharmacoepidemiology, ${ }^{1}$ for the EUROCAT Antiepileptic Study Working Group
\end{abstract}

Department of

PharmacoEpidemiology and PharmacoEconomics, Division of Pharmacy, University of Groningen Netherlands

${ }^{2}$ EUROCAT Central Registry

Institute of Nursing Research and School of Nursing, University of Ulster, Antrim, UK

${ }^{3}$ Wolfson Institute of Preventive Medicine, Barts and the London School of Medicine and Dentistry, Queen Mary University of London, London, UK

${ }^{4}$ Wessex Clinical Genetics Service, Southampton University Hospitals Trust, Southampton, UK

${ }^{5}$ Lillebaelt Hospital, Kolding, Denmark

Correspondence to: $\mathrm{L}$ de Jong-van den Berg

l.t.w.de.jong-van.den.berg@rug.nl

Cite this as: $B M J$ 2010;341:C6581 doi:10.1136/bmi.c6581

\section{ABSTRACT}

Objective To identify specific major congenital malformations associated with use of carbamazepine in the first trimester of pregnancy.

Design A review of all published cohort studies to identify key indications and a population based case-control study to test these indications.

Setting Review of PubMed, Web of Science, and Embase for papers about carbamazepine exposure in the first trimester of pregnancy and specific malformations, and the EUROCAT Antiepileptic Study Database, including data from 19 European population based congenital anomaly registries, 1995-2005.

Participants The literature review covered eight cohort studies of 2680 pregnancies with carbamazepine monotherapy exposure, and the EUROCAT dataset included 98075 registrations of malformations covering over 3.8 million births.

Main outcome measures Overall prevalence for a major congenital malformation after exposure to

carbamazepine monotherapy in the first trimester. Odds ratios for malformations with exposure to carbamazepine among cases (five types of malformation identified in the literature review) compared with two groups of controls: other non-chromosomal registrations of malformations and chromosomal syndromes.

Results The literature review yielded an overall prevalence for a major congenital malformation of 3.3\% (95\% confidence interval 2.7 to 4.2 ) after exposure to carbamazepine monotherapy in the first trimester. In 131 registrations of malformations, the fetus had been exposed to carbamazepine monotherapy. Spina bifida was the only specific major congenital malformation significantly associated with exposure to carbamazepine monotherapy (odds ratio 2.6 (95\% confidence interval 1.2 to 5.3) compared with no antiepileptic drug), but the risk was smaller for carbamazepine than for valproic acid $(0.2,0.1$ to 0.6$)$. There was no evidence for an association with total anomalous pulmonary venous return (no cases with carbamazepine exposure), cleft lip (with or without palate) (0.2, 0.0 to 1.3), diaphragmatic hernia (0.9, 0.1 to 6.6), or hypospadias ( $0.7,0.3$ to 1.6$)$ compared with no exposure to antiepileptic drugs. Further exploratory analysis suggested a higher risk of single ventricle and atrioventricular septal defect.

Conclusion Carbamazepine teratogenicity is relatively specific to spina bifida, though the risk is less than with valproic acid. Despite the large dataset, there was not enough power to detect moderate risks for some rare major congenital malformations.

\section{INTRODUCTION}

Carbamazepine is one of the most commonly used antiepileptic drugs in Europe among women of childbearing age. Several cohort studies have evaluated the risk of major congenital malformations associated with carbamazepine, and it seemed to be less teratogenic than valproic acid. ${ }^{1-6}$ Although around 3000 pregnancies with recorded carbamazepine exposure have been described in the literature, each individual study on its own is too small to have the statistical power to detect risks for specific congenital malformations compared with other antiepileptic drugs. ${ }^{48}$

The EUROCAT (European Surveillance of Congenital Anomalies) Antiepileptic Study Database, which was set up for a study of lamotrigine, covered 3881592 births and contains 131 registrations of malformation in pregnancy outcomes exposed to carbamazepine monotherapy, more than in any other published study. ${ }^{9}$ This enabled the examination of risks of specific major congenital malformations associated with carbamazepine monotherapy.

We reviewed and combined studies on carbamazepine to identify indications of increased risks of specific malformations after intrauterine exposure to carbamazepine monotherapy in the first trimester of pregnancy. We tested these indications, or prior hypotheses, in a case-control study using two control groups of malformations. 
Table 1| Overview of included cohort studies on effects of carbamazepine taken during pregnancy on congenital malformations

\begin{tabular}{|c|c|c|c|c|c|}
\hline \multirow[b]{2}{*}{ Study } & \multirow[b]{2}{*}{ Country } & \multirow[b]{2}{*}{ Included years } & \multicolumn{3}{|c|}{ Exposed to carbamazepine monotherapy } \\
\hline & & & Number & Malformed & Rate $(95 \% \mathrm{Cl})$ \\
\hline Samrén $1997^{14}$ & $\begin{array}{l}\text { Berlin and Magdeburg, Germany; Helsinki, Finland; } \\
\text { Rotterdam, Netherlands; epilepsy institutes }\end{array}$ & $1972-90$ & 280 & 22 & $7.9(5.2$ to 11.6$)$ \\
\hline Diav-Citrin $2001^{11}$ & Israeli Teratogen Information Service & Jan 1989-March 1999 & 108 & 6 & $5.6(2.6$ to 11.8$)$ \\
\hline Kaaja $2003^{12}$ & Finland, Helsinki & Jan 1980-Sep 1998 & 363 & 10 & $2.8(1.5$ to 5.0$)$ \\
\hline Sabers $2004^{13}$ & 6 hospitals, Denmark, & Sep 1996-May 2000 & 18 & 0 & $0(0.0$ to 17.6$)$ \\
\hline Wide $2004^{5}$ & Sweden & Jul 1995-Dec 2001 & 703 & 28 & $4.0(2.8$ to 5.7$)$ \\
\hline Meador $2006^{2}$ & 25 epilepsy centres, UK and US & Oct 1999-Feb 2004 & 110 & 5 & 4.5 (2.0 to 10.2$)$ \\
\hline Morrow $2006^{3}$ & Pregnancy registry, UK & Dec 1996-March 2005 & 900 & 20 & $2.2(1.4$ to 3.4$)$ \\
\hline Vajda $2007^{4}$ & Pregnancy registry, Australia & Jul 1999-Oct 2002 & 198 & 10 & $5.1(2.8$ to 9.0$)$ \\
\hline Total & - & $1972-2005$ & 2680 & 101 & 3.8 (3.1 to 4.6$)$ \\
\hline $\begin{array}{l}\text { Major according to } \\
\text { EUROCAT* }^{\star}\end{array}$ & - & - & 2680 & 89 & $3.3(2.7$ to 4.2$)$ \\
\hline
\end{tabular}

*According to EUROCAT major congenital malformation classification (based on ICD-10) 12 were only minor malformations and were therefore excluded.

\section{METHODS}

EUROCAT

The EUROCAT Antiepileptic Study Database has been previously described. ${ }^{6910}$ It was drawn from 19 population based registries of congenital anomaly in Europe, covering 3881592 births in Europe in 19952005 and 98075 major congenital malformations: 86291 non-chromosomal and 11784 chromosomal. Information was available for live births, still births or late fetal deaths from 20 weeks' gestation, and terminations of pregnancy after prenatal diagnosis. The database includes data from registries in Belgium (Antwerp and Hainaut), Croatia (Zagreb), Denmark (Odense), France (Paris and Strasbourg), Germany (Mainz and Saxony-Anhalt), Ireland (Cork and Kerry), Italy (Emilia Romagna and Tuscany), Malta, Netherlands (northern part), Norway, Poland (Wielkopolska, rest of Poland), Spain (Basque Country), Switzerland (Vaud), and the United Kingdom (Wales).

\section{Literature review}

We reviewed PubMed, Web of Science, and Embase for papers about carbamazepine exposure in the first trimester of pregnancy and specific malformations using the following search strategy: ((“Carbamazepine"[Mesh] OR "antiepileptic drugs"[ti] OR "AED"[ti]) AND ("Congenital Abnormalities"[Majr] OR "Pregnancy Complications/drug therapy"[Majr] OR "birth defects"[ti]) NOT "Clinical Trials, Phase I as Topic"[Mesh] NOT ("Models, Animal"[Mesh] OR "Animal Experimentation"[Mesh])) AND "Cohort Studies"[Mesh] (PubMed n=44) and "carbamazepine and malformation and pregnancy' (Web of Science $\mathrm{n}=141$ and Embase $\mathrm{n}=30$ ). We identified nine cohort studies that contained a specified case list of all pregnancy outcomes with malformation (table 1). ${ }^{1-511-14}$ We contacted four authors to get more information about the case list; three were able to supply the information requested and were included, ${ }^{13-5}$ the fourth was excluded. ${ }^{1}$

The eight cohort studies included outcomes of 2680 pregnancies with carbamazepine monotherapy exposure. Of these, 101 babies had a malformation, 89 of which were classified as major according to EUROCAT $^{10}$ and were classified according to 49 standard EUROCAT congenital anomaly subgroups. A case could be counted only once in each subgroup but could be counted in more than one subgroup. The 12 cases classified as only minor malformations according to the EUROCAT classification were excluded. ${ }^{10}$ Based on these eight cohorts, the overall prevalence for a major congenital malformation after exposure to carbamazepine monotherapy in the first trimester was 3.3\% (95\% confidence interval 2.7 to 4.2) (table 1).

The prevalence for specific subgroups of congenital anomaly was calculated by dividing the subgroup totals by the total outcomes in pregnancies with carbamazepine monotherapy exposure. This prevalence was compared with the prevalence in the population covered by the EUROCAT Antiepileptic Study Database (excluding pregnancies with exposure to antiepileptic drugs), with $\chi^{2}$ test with Yate's correction in S-PLUS 7.0. In the combined literature cohort five subgroups had a significantly higher prevalence than expected $(\mathrm{P}<0.05)$ (table 2) and were considered "indications" to be tested in the case-control study: spina bifida, total anomalous pulmonary venous return, cleft lip (with or without palate), diaphragmatic hernia, and hypospadias.

To check if we missed any indication by concentrating on published cohort studies in the literature review, we searched for additional indications in abstracts of case-control studies. We found one additional indication for the risk of cleft palate. ${ }^{15} \mathrm{We}$ excluded cleft palate malformations from our study control group and examined these registrations separately. Indications from other case-control studies had all been identified in the review of cohort studies.

\section{Case-control study}

We carried out a population based case-control study to test the five indications identified in the literature review. We compared the odds of exposure to 
Table $2 \mid$ Result of review of eight cohort studies. All specific malformations found in literature review were classified to 75 malformation subgroups; 49 subgroups had at least one case and five (shown here) showed significant increased prevalence compared with EUROCAT population. Figures are numbers of cases (prevalence per 1000 registrations)

\begin{tabular}{lccc} 
& $\begin{array}{c}\text { Literature } \\
(\mathrm{n}=2680)\end{array}$ & $\begin{array}{c}\text { EUROCAT* } \\
(\mathrm{n}=3 \text { 869 947) }\end{array}$ & P value† \\
Anomalous pulmonary venous return & $2(0.75)$ & $134(0.03)$ & $<0.001$ \\
\hline Cleft lip, with or without palate & $7(2.61)$ & $3634(0.94)$ & 0.012 \\
\hline Diaphragmatic hernia & $3(1.12)$ & $766(0.20)$ & 0.007 \\
\hline Hypospadias & $12(4.48)$ & $5418(1.40)$ & $<0.001$ \\
\hline Spina bifida & $6(2.24)$ & $1933(0.50)$ & $<0.001$
\end{tabular}

*Excluding registrations of chromosomal malformations and malformations with reported exposure to antiepileptic drugs.

†Calculated with $x^{2}$ test with Yates's correction. names were known. In 95\% of all registrations with carbamazepine exposure, there was a diagnosis of maternal epilepsy. To avoid misclassification of exposure we excluded all registrations with an associated reported diagnosis of maternal epilepsy but without maternal use of antiepileptic drugs in the first trimester (42 cases: spina bifida (8), cleft lip (12), diaphragmatic hernia (5), and hypospadias (17); and 195 controls). We compared carbamazepine monotherapy in the first trimester of pregnancy with "no antiepileptic drug exposure," "valproic acid monotherapy," and "other antiepileptic drug monotherapy excluding valproic acid." In the comparison with valproic acid monotherapy we excluded from the control group malformations associated with valproic acid exposure: atrial septal defect, polydactyly, and craniosynostosis. ${ }^{6}$

carbamazepine monotherapy among each of these five malformations under study (cases) with the odds of exposure among two control groups of malformations: a non-chromosomal and a chromosomal control group. As valproic acid has been shown to be more teratogenic than other antiepileptic drugs, we compared carbamazepine monotherapy exposure with "no antiepileptic drug exposure," "valproic acid monotherapy," and "other antiepileptic drug monotherapy" (excluding valproic acid). ${ }^{6}$

\section{Case definition}

Cases were defined as all live births, fetal deaths from 20 weeks' gestation, and terminations of pregnancy after prenatal diagnosis, non-chromosomal and nonmonogenic, with at least one of the following major congenital malformations: spina bifida, total anomalous pulmonary venous return, cleft lip (with or without palate), diaphragmatic hernia, and hypospadias. We excluded all cases of diagnosed monogenic syndrome $(\mathrm{n}=180)$.

\section{Control definition}

Control group 1 included live births, fetal deaths from 20 weeks' gestation, and terminations of pregnancy after prenatal diagnosis that involved major malformations other than the five malformations under study. We excluded chromosomal syndromes as well as registrations with cleft palate or Pierre Robin sequence $(n=2320)$ and all anencephaly or encephalocele $(\mathrm{n}=1860)$ to avoid possible misclassification from an aetiologically similar diagnosis. Five controls were excluded because of unknown type of birth.

Control group 2 included live births, fetal deaths from 20 weeks' gestation, and terminations of pregnancy after prenatal diagnosis with chromosomal syndromes. Two controls in this group were excluded because of unknown type of birth.

\section{Exposure}

All registrations with associated maternal use of antiepileptic drugs or maternal epilepsy, or both, were selected, verified by the local registry, and coded by ATC (Anatomical Therapeutic Chemical Classification) code. ${ }^{16}$ After verification over $99 \%$ of all drug

\section{Statistical analyses}

Odds ratios were calculated with logistic regression in Stata. Crude odds ratios were calculated without correction for any possible confounder and including all registries even if they had no registration with carbamazepine exposure (see appendix 1 on bmj.com). Odds ratios were adjusted for maternal age (categorised into $<25,25-29,30-34$, and $>34$ years) and year of birth of the child (categorised as before 1999, 1999-2001, and 2002 onwards). If there were sufficient numbers of cases the adjusted odds ratio was also corrected for the reporting registry (registries with no carbamazepine exposure were excluded). No systematically recorded information on other potentially important confounders was available in our dataset.

We also conducted an exploratory analysis to check if there was evidence in the data of specific malformations related to carbamazepine monotherapy that were not identified by the literature review. We compared the proportion of each subgroup of specific non-chromosomal congenital anomalies (excluding the five case groups, but reincluding cleft palate, anencephaly, and encephalocele) for malformations with carbamazepine monotherapy exposure with all non-chromosomal EUROCAT registrations without reported epilepsy or use of antiepileptic drugs. We compared differences between proportions with a $\chi^{2}$ test.

\section{RESULTS}

In the included study population 516 registered malformations were in pregnancy outcomes with recorded exposure to any antiepileptic drug in the first trimester of pregnancy, 5.5 per 1000 registrations (516/93 436). The exposure to antiepileptic drugs was more than twice as high among the cases (10.9 per 1000 registrations) than among the controls (5.0 and 3.2 per 1000 registrations) (table 3 ). This difference between cases and controls was not seen in registered malformations with any recorded exposure to carbamazepine monotherapy. The exposure to carbamazepine monotherapy was comparable among cases $(1.4 / 1000)$ and among non-chromosomal controls (1.5/1000). The exposure among chromosomal controls was lower $(0.9 / 1000)$. The exposure to valproic acid 
Table 3 |Exposure to antiepileptic drugs during pregnancy recorded among registrations of congenital malformations (cases and controls). Figures are numbers of cases (prevalence per 1000 registrations)

\begin{tabular}{|c|c|c|c|}
\hline & $\begin{array}{l}\text { Non-chromosomal cases } \\
\text { under study* }(n=11790)\end{array}$ & $\begin{array}{l}\text { Control group } 1 \dagger \\
\qquad(n=69883)\end{array}$ & $\begin{array}{l}\text { Control group } 2 \ddagger \\
(n=11763)\end{array}$ \\
\hline Any antiepileptic drug & 129 (10.9) & $349(5.0)$ & $38(3.2)$ \\
\hline Any antiepileptic drug monotherapy & $104(8.8)$ & $282(4.0)$ & $32(2.7)$ \\
\hline Valproic acid monotherapy & $66(5.6)$ & $102(1.5)$ & $13(1.1)$ \\
\hline Carbamazepine monotherapy & $16(1.4)$ & $105(1.5)$ & $10(0.9)$ \\
\hline Other monotherapy & $22 \S(1.9)$ & $75 \mathbb{1}(1.1)$ & $9^{\star \star}(0.8)$ \\
\hline
\end{tabular}

*All cases with anomalous pulmonary venous return, cleft lip (with or without palate), diaphragmatic hernia, hypospadias, or spina bifida.

†Non-chromosomal malformations, excluding malformations under study.

$\ddagger$ All chromosomal malformations.

§Phenobarbital (9), lamotrigine (5), clonazepam (3), levetriacetam (2), unspecified antiepileptic drugs (3).

TLamotrigine (33), phenobarbital (15), oxcarbazepine (10), phenytoin (5), clonazepam (3), primidon (3),

methylphenobarbital (2), topiramate (2), ethosuximide (1), unspecified (1).

**Phenobarbital (4), lamotrigine (2), clonazepam (1), oxcarbamazepine (1), phenytoin (1).

monotherapy was higher among cases than among both control groups (table 3).

The five case subgroups included 11790 cases (table 4). Eighty two cases were included in two different subgroups. The control groups consisted of 69883 non-chromosomal malformed registrations and 11763 chromosomal registrations.

\section{Carbamazepine monotherapy $v$ no antiepileptic drugs}

In the comparison of carbamazepine monotherapy exposure with no antiepileptic drugs, one of the five indications found in the literature was confirmed: the odds ratio for spina bifida was 2.6 (95\% confidence interval 1.2 to 5.3 ) compared with non-chromosomal controls and 4.2 (1.5 to 11.2 ) compared with chromosomal controls. The odds ratios for cleft lip (with or without palate), diaphragmatic hernia, and hypospadias were not appreciably increased; they were all around or below 1, though for diaphragmatic hernia the confidence interval was wide. We could not perform meaningful analyses for total anomalous pulmonary venous return because we did not have any cases with exposure to carbamazepine (table 4).

\section{Carbamazepine monotherapy $v$ other antiepileptic drug monotherapy}

In contrast with the comparison with "no antiepileptic drugs" the exposure to carbamazepine monotherapy resulted in a reduced risk for spina bifida compared with valproic acid monotherapy, which was significant in the comparison with control group 1 (0.2, 0.1 to 0.6 ). Compared with other antiepileptic drug monotherapy excluding valproic acid, exposure to carbamazepine monotherapy showed no difference in the risk for spina bifida (1.1, 0.4 to 3.6).

For hypospadias we found a significantly lower risk for carbamazepine monotherapy than for valproic acid monotherapy $(0.2,0.1$ to 0.5$)$ and again no difference in risk in comparison with other antiepileptic drug monotherapy excluding valproic acid $(0.8,0.2$ to 2.9$)$.

The risk for cleft lip with or without palate was significantly lower for carbamazepine monotherapy than for other antiepileptic drug monotherapy excluding valproic acid $(0.1,0.0$ to 0.6$)$ (over half of these cases had recorded exposure to phenobarbital). No significant difference was seen for carbamazepine monotherapy compared with valproic acid monotherapy $(0.3$, 0.0 to 2.6 ), but the point estimate was decreased. The results for diaphragmatic hernia also showed decreased odds ratios but with wide confidence intervals (table 4).

We tested the one additional indication identified in literature, cleft palate, in the EUROCAT dataset, but we did not confirm this indication compared with no antiepileptic drug exposure (crude odds ratio 1.3 (0.4 to 4.1 ) with non-chromosomal controls). ${ }^{15}$

\section{Exploratory analysis}

The exploratory analysis of all malformation subgroups found that all proportions were similar (P>0.05) except for two subgroups: single ventricle $(2.3 \% \quad(\mathrm{n}=3)$ of carbamazepine exposed $v \quad 0.3 \%$ expected, $\left.\chi^{2} \mathrm{P}<0.001\right)$ and atrioventricular septal defect $(3.1 \%(\mathrm{n}=4)$ of carbamazepine exposed $v 0.8 \%$ expected, $\chi^{2} \mathrm{P}<0.011$, data available on request). When we removed registrations with these malformations from our non-chromosomal control group, our results regarding the five indication case groups stayed essentially the same.

\section{DISCUSSION}

\section{Interpretation of the results}

Of the five indications for specific malformations associated with exposure to carbamazepine monotherapy that we identified from published cohort studies, spina bifida was the only confirmed indication (odds ratio 2.6 (1.2 to 5.3) for comparison with no exposure to antiepileptic drug and with the non-chromosomal controls). All other case groups resulted in an odds ratio around or below 1 . According to the upper confidence limits, we could exclude $50 \%$ of excess risks for cleft lip and hypospadias with some degree of certainty, but further surveillance would be necessary to gain larger numbers to estimate risks for diaphragmatic hernia and total anomalous pulmonary venous return with more precision.

In our analyses comparing exposure to carbamazepine monotherapy with valproic acid monotherapy we found a significantly decreased risk for spina bifida (odds ratio $0.2,0.1$ to 0.6 ) and hypospadias (odds ratio $0.2,0.1$ to 0.5$)$, indicating that the risk of spina bifida and hypospadias with carbamazepine is less than with valproic acid. In our study of valproic acid 6 we found a sixfold risk for spina bifida and a sevenfold risk for hypospadias with valproic acid monotherapy compared with exposure to another antiepileptic drug with the non-chromosomal control group.

It is noteworthy that the risk for cleft lip with or without palate was significantly more related to other antiepileptic drug monotherapy (excluding valproic acid) than to carbamazepine monotherapy (odds ratio 0.1 , 0.0 to 0.6 ). Over half of these cases were exposed to 
Table $4 \mid$ Adjusted* odds ratios ( $95 \%$ confidence intervals) for exposure to carbamazepine monotherapy compared with no antiepileptic drug, valproic acid, and other antiepileptic drugs, with two control groups: non-chromosomal malformations (control 1) and chromosomal malformations (control 2)

\begin{tabular}{|c|c|c|c|c|c|c|c|}
\hline \multirow[b]{2}{*}{ Malformation subgroup (No of cases) } & \multirow{2}{*}{$\begin{array}{l}\text { Carbamaze- } \\
\text { pine }\end{array}$} & \multicolumn{2}{|c|}{ No antiepileptic drug } & \multicolumn{2}{|c|}{ Valproic acid monotherapy $\dagger$} & \multicolumn{2}{|c|}{ Other monotherapy $\ddagger$} \\
\hline & & Control 1 & Control 2 & Control 1 & Control 2 & Control 1 & Control 2 \\
\hline Spina bifida $(n=2048)$ & 8 & $2.6(1.2$ to 5.3$)$ & 4.2 (1.5 to 11.2$)$ & $0.2(0.1$ to 0.6$)$ & $0.3(0.1$ to 1.2$)$ & $1.1(0.4$ to 3.6$)$ & $1.4(0.3$ to 6.6$)$ \\
\hline Total anomalous pulmonary venous return $(n=132)$ & 0 & - & - & - & - & - & - \\
\hline Cleft lip (with or without palate) $(n=3544)$ & 1 & $0.2(0.0$ to 1.3$)$ & $0.2(0.0$ to 1.7$)$ & 0.3 (0.0 to 2.6$)$ & 0.2 (0.0 to 2.7$)$ & $0.1(0.0$ to 0.6$)$ & 0.0 (0.0 to 0.5$)$ \\
\hline Diaphragmatic hernia $(n=755)$ & 1 & $0.9(0.1$ to 6.6$)$ & $1.0(0.1$ to 8.5$)$ & $0.5(0.0$ to 4.5$)$ & $0.4(0.0$ to 5.8$)$ & $0.2(0.0$ to 2.2$)$ & $0.2(0.0$ to 2.5$)$ \\
\hline Hypospadias, boys only $(n=5393)$ & 6 & $0.7(0.3$ to 1.6$)$ & $0.5(0.2$ to 1.8$)$ & $0.2(0.1$ to 0.5$)$ & $0.1(0.0$ to 0.7$)$ & $0.8(0.2$ to 2.9$)$ & $0.4(0.1$ to 4.0$)$ \\
\hline
\end{tabular}

*Adjusted for year of birth and maternal age. Odds ratios for comparison with no antiepileptic drug additionally adjusted for reporting centre.

†Excluding malformations associated with valproic acid exposure. Cases exposed to valproic acid: spina bifida (27), total anomalous pulmonary venous return (2), cleft lip (3), diaphragmatic hernia (2), hypospadias (32).

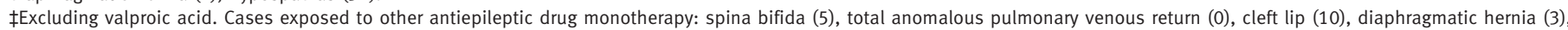
hypospadias (5).

phenobarbital, which is known to be associated with cleft lip and palate. ${ }^{17}$

\section{Comparison with other studies}

The rate of major malformation with exposure to carbamazepine that we found when combining the eight cohort studies (3.3\%) was lower than the rate reported in the only meta-analysis in the literature $(44 / 797$; $5.5 \%, 4.1 \%$ to $7.3 \%) .{ }^{18}$ Eleven of the 16 studies included in the meta-analysis contained information about malformations. Three of these 11 presented a case list with specific malformations and were included in our literature review. The difference in the rate of malformations could be explained by dissimilarities in classification of minor malformations for exclusion.

In a general review of the literature, we did not find any strong suggestions of specific malformations other than our five case groups, except for a recently published abstract based on the North American AED Pregnancy Registry, which suggested a 24 -fold (7.9 to 74.4) increase in the rate of isolated cleft palate with carbamazepine. ${ }^{15}$ In the EUROCAT dataset we did not confirm this strong indication, either in the proportional exploratory analysis or by calculation of odds ratios.

\section{Strengths and limitations of the study}

One limitation of our study is that we used controls with malformations. Controls with non-chromosomal malformations will result in an underestimation of the effect if there are any individuals left in the control group with malformations that are related to the exposure. Our exploratory analysis was designed to see if there were specific malformations of concern; we found single ventricle and atrioventricular septal defect to represent a higher proportion than expected (though this might be a chance finding associated with multiple comparisons). When we excluded these two malformations from the control group, our results stayed essentially the same. Chromosomal controls can lead to an overestimation of effect of drug exposure if exposure is not recorded completely because of the lack of relevance of drug exposure in early pregnancy. We have examined this in previous studies of lamotrigine and valproic acid ${ }^{69}$ and found no evidence of substantially poorer recording of exposure to antiepileptic drugs for chromosomal controls, either by examination of exposure rates or by examination of information gathering procedures for exposure. Exposure information is mainly collected prospectively in medical records and is ascertained by the registry regardless of type of malformation. In a previous study evaluating exposure to valproic acid and specific congenital malformations, we found similar odds ratios for non-chromosomal and chromosomal controls, while in our study of lamotrigine exposure and orofacial clefts, as in the current carbamazepine study, we found greater odds ratios with chromosomal controls than with non-chromosomal control group. One interpretation is that there is a generalised risk of malformation associated with carbamazepine, which is not specific to a few malformation groups. With that interpretation, the difference in exposure rate between the control groups would suggest an up to $50 \%$ generalised increase risk of major malformations with carbamazepine. Another interpretation is that carbamazepine "protects" against chromosomal malformations-for example, by raising the chance of an early miscarriage for affected pregnancies. In relation to the finding regarding spina bifida, we consider that the true odds ratio is likely to be between the estimates obtained from the two control groups.

The apparent specificity of effect of carbamazepine for spina bifida, compared with valproic acid, with a much larger range of effects, might be a useful biological clue in elucidating the underlying teratogenic mechanism. It is also possible that spina bifida is in part related to the underlying epilepsy rather than the drug used, though our results show that the drug used at least affects the level of risk of spina bifida. In this large population based study, we carried out a direct comparison between the risks for specific malformations associated with carbamazepine monotherapy compared with valproic acid monotherapy. A limitation in our comparison of risks between exposure to different types of antiepileptic drug was that we did not have the information to adjust for type of epilepsy, frequency of seizures, used of folic acid, and dose of the antiepileptic drug.

\section{Conclusions and policy implications}

Although most antiepileptic drugs taken during pregnancy significantly increase the risk for one or more 


\section{WHAT IS ALREADY KNOWN ON THIS TOPIC}

Women of childbearing age with epilepsy need to make decisions about the safest drug to use well before pregnancy

Carbamazepine exposure in the first trimester increases the risk for major congenital malformations

Most individual studies have been too small to clarify which specific malformations are associated with carbamazepine exposure or to directly compare risks with other antiepileptic drugs

\section{WHAT THIS STUDY ADDS}

Spina bifida was significantly associated with exposure to carbamazepine monotherapy compared with no use of antiepileptic drugs, but the risk was significantly smaller than for valproic acid

Associations between carbamazepine and the four other malformations examined (total anomalous pulmonary venous return (no exposed cases), cleft lip with or without palate, diaphragmatic hernia, or hypospadias) were not confirmed

specific fetal malformations, the occurrence of these malformations is nevertheless rare. Most exposed pregnancies result in a baby without malformation. The best option regarding antiepileptic drug treatment can be chosen only on an individual basis by the woman and neurologist before pregnancy, weighing the benefits of epilepsy control against the risk of teratogenicity. In this study we have confirmed that carbamazepine is less teratogenic than valproic acid. A Cochrane review found no evidence to support the belief that valproic acid is superior to carbamazepine for generalised tonic-clonic seizures. ${ }^{19}$ Therefore, we agree with the recent recommendation of the American Academy of Neurology to avoid valproic acid in pregnancy if possible. ${ }^{20}$ Our literature review gives a $3.3 \%$ risk of major malformations with carbamazepine monotherapy, and our case-control study shows that the major concern is a moderately increased risk of spina bifida. This should to help in decision regarding whether carbamazepine should be the antiepileptic drug of choice in pregnancy.

We thank J Morrow, U Kini, F Vajda, and K Wide for providing us with more detailed information about the cases described in their papers ${ }^{13.5}$ and I Barisic for case review.

EUROCAT Antiepileptic Study Working Group: Christine VerellenDumoulin (Centre de Génétique Humaine IPG), Vera Nelen (Provinciaal Instituut voor Hygiene), Belgium; Ingeborg Barisic (Children's University Hospital Zagreb, Croatia); Ester Garne (Lillebaelt Hospital, Kolding, Denmark); Babak Khoshnood (Institut National de la Sante et de la Recherche Medicale, INSERM) Bérénice Doray (Registre des

Malformations Congenitales D'Alsace), France; Simone Poetzsch (Ottovon-Guericke Universitat Megdeburg), Awi Wiesel (Johannes Gutenberg Universitat, Genurtenregister Mainzer Modell), Germany; Mary O'Mahony (Health Service Executive, Ireland); Anna Pierini (Istituto di Fisiologia Clinica del Consiglio Nazionale delle Ricerche), Francesca Rivieri (Azienda Ospedaliero Universitaria di Ferrara), Italy; Miriam Gatt (Department of Health Information and Research, Malta); Marian Bakker (University Medical Centre Groningen, University of Groningen, Netherlands); Kar Melve (Norwegian Institute of Public Health, Medical Birth Registry of Norway); Anna Latos-Bielenska, Jan P Mejnartowicz (Uniwersytet Medyczny im. Karola Marcinkowskiego w Poznaniu, Poland); Isabel Portillo (Direccion Salud Publica, Departamento Sanidad, Gobierno Vasco, Spain); Marie-Claude Addor (Registre Vaudois des Malformations, Switzerland); David Tucker (Swansea NHS Trust, Congenital Anomaly Register and Information Service for Wales).
Contributors: JJ, HD, and LJvB drafted the protocol and paper. LJvB, JJ, and ML coordinated the study. JJ conducted the literature review, conducted validation and coding of drug exposure information, and constructed the tables. ML and JJ compiled the study database. JM conducted the statistical analysis. All authors corrected all versions of the paper. EG, DW, and IB classified malformed cases. EG and IB and all other members of the EUROCAT Antiepileptic Drug Exposure Working Group not mentioned above contributed data to the study from their registry, checked the accuracy of drug exposure information, checked data tables, and commented on the first and final drafts. $\mathrm{HD}$ and LJvB are guarantors. Funding: The EUROCAT Central Database is supported in part by the European Public Health Programme, with various sources of public funding for individual registries. Additional funding was obtained from GlaxoSmithKline for a study of lamotrigine, during which the antiepileptic study database was constructed (all authors were involved in this study). GlaxoSmithKline was not involved in the present study.

Competing interests: All authors have completed the Unified Competing Interest form at www.icmje.org/coi_disclosure.pdf (available on request from the corresponding author) and declare: no personal support from any organisation for the submitted work; no financial relationships with any organisations that might have an interest in the submitted work in the previous 3 years; no other relationships or activities that could appear to have influenced the submitted work.

Ethical approval: Not required

Data sharing: No additional data available.

1 Kini U, Adab N, Vinten J, Fryer A, Clayton-Smith J, Liverpool and Manchester Neurodevelopmental Study Group. Dysmorphic features: an important clue to the diagnosis and severity of fetal anticonvulsant syndromes. Arch Dis Child Fetal Neonatal Ed 2006;91:90-5.

2 Meador KJ, Baker GA, Finnell RH, Kalayjian LA, Liporace JD, Loring DW, et al. In utero antiepileptic drug exposure, fetal death and malformations. Neurology 2006;67:407-12.

3 Morrow J, Russell A, Guthrie E, Parsons L, Robertson I, Waddell R, et al. Malformation risks of antiepileptic drugs in pregnancy: a prospective study from the UK Epilepsy and Pregnancy Register. J Neurol Neurosurg Psychiatry 2006;77:193-8.

4 Vajda FJE, Lander CM, Hitchcock A, Graham J, Solinas C, O'Brien T, et al. Changing Australian prescribing patterns for antiepileptic drugs in pregnancy and their possible consequences. J Clin Neurosci 2007;14:611-7.

5 Wide K, Winbladh B, Källén B. Major malformations in infants exposed to antiepileptic drugs in utero, with emphasis on carbamazepine and valproic acid: a nation-wide, population-based register study. Acta Pædiatr 2004;93:174-6.

6 Jentink J, Loane MA, Dolk H, Barisic I, Garne E, Morris JK, et al, for the EUROCAT Antiepileptic Study Working Group. Valproic acid monotherapy in pregnancy and major congenital malformations. $N$ Engl J Med 2010;362:2185-93.

7 Perucca E. Birth defects after prenatal exposure to antiepileptic drugs. Lancet Neurol 2005;4:781-6.

8 Tomson T, Perucca E, Battino D. Navigating toward fetal and materna health: the challenge of treating epilepsy in pregnancy. Epilepsia 2004; 45:1171-5.

9 Dolk H, Jentink J, Loane MA, Morris JK, De Jong-van den Berg LTW, the EUROCAT Antiepileptic Drug Working Group. Does lamotrigine use in pregnancy increase orofacial cleft risk relative to other malformations? Neurology 2008;71:714-22.

10 EUROCAT. EUROCAT Guide 1.3 and reference documents. Instructions for the registration and surveillance of congenital anomalies. 2005. www.eurocat-network.eu/content/EUROCATGuide-1.3.pdf.

11 Diav-Citrin O, Shechtman S, Arnon J, Ornoy A. Is carbamazepine teratogenic? A prospective controlled study of 210 pregnancies. Neurology 2001;57:321-4

12 Kaaja E, Kaaja R, Hiilesmaa V. Major malformations in offspring of women with epilepsy. Neurology 2003;60:575-9.

13 Sabers A, Dam M, a-Rogvi-Hansen B, Boas J, Sidenius P, Laue Friis M, et al. Epilepsy and pregnancy: lamotrigine as main drug used. Acta Neurol Scand 2004;109:9-13.

14 Samrén EB, Van Duijn CM, Koch S, Hiilesmaa VK, Klepel H, Bardy AH, et al. Maternal use of antiepileptic drugs and the risk of major congenital malformations: a joint European prospective study of human teratogenesis associated with maternal epilepsy. Epilepsia 1997;38:981-90.

15 Hernandez-Diaz S, Smith CR, Wyszynski DF, Holmes LB. Major malformations among infants exposed to carbamazepine during pregnancy (Teratology Society Abstracts). Birth Defects Res A Clin Mol 2007;79:357. 
16 World Health Organization. ATC index with DDDs 2003. Guidelines for ATC classification and DDD assignment 2003. Version 13. WHO Collaborating Centre for Drug Statistics Methodology, 2003.

17 Ornoy A. Neuroteratogens in man: an overview with special emphasis on the teratogenicity of antiepileptic drugs in pregnancy. Reprod Toxicol 2006;22:214-26.

18 Matalon S, Schechtman S, Goldzweig G, Ornoy A. The teratogenic effect of carbamazepine: a meta-analysis of 1255 exposures. Reprod Toxicol 2002;16:9-17.
19 Marson AG, Williamson PR, Hutton JL, Clough HE, Chadwick DW. Carbamazepine versus valproate monotherapy for epilepsy. Cochrane Database Syst Rev 2000;3:CD001030.

20 Harden CL, Meador KJ, Pennell PB, Hauser WA, Gronseth GS, French JA, et al. Management issues for women with epilepsy-focus on pregnancy (an evidence-based review): II. Teratogenesis and perinatal outcomes. Epilepsia 2009;50:1237-46.

Accepted: 27 September 2010 